\title{
基于 GPU 的长码直接捕获方法
}

\author{
牟卫华 $1,2^{*}$ ，唐小妹 ${ }^{1}$ ，马春江 ${ }^{1}$ ，欧钢 ${ }^{1,2}$ ，王飞雪 ${ }^{1,2}$ \\ 1. 国防科学技术大学电子科学与工程学院, 长沙 410073 \\ 2. 北斗卫星导航产品 2301 质量检测中心, 长沙 410073 \\ * 通信作者. E-mail: drmou@163.com
}

收稿日期: 2016-08-22；接受日期: 2017-01-20; 网络出版日期: 2017-07-06 国家自然科学基金 (批准号: 61601485) 资助项目

摘要 针对长周期伪码扩频信号的捕获问题, 分析了局部相关的码相位空间捕获模型和 FFT 实现 方法. 为了能够对数据处理任务进行分割处理, 充分利用 GPU 加速 FFT 运算, 本文研究了部分重 叠局部相关长码直接捕获算法, 提出了基于 GPU 的全局直接搜索和分段重叠搜索长码直接捕获方 法. 该方法利用 FFT 并行搜索伪码相位, 在 GPU 中批处理加速 FFT 运算. 实验结果表明, 相比 $\mathrm{CPU}$ 实现的方法, 基于 GPU 的长码直接捕获方法显著提高了捕获速度.

关键词图形处理单元, 并行计算, 长码, 直接捕获, 快速 Fourier 变换

\section{1 引言}

长周期伪码扩频信号抗干扰性能相比短码更强, 在短码受干扰条件下, 只能依赖长码的直接捕获, 因此长码直接捕获技术是导航领域的一个研究热点. 伪码扩频信号捕获阶段需要搜索确定伪码相位 和 Doppler 初始值, 长码直接捕获需要搜索的码相位不确定范围相比短码更大, 通常是短码搜索范围 的 $10^{3} \sim 10^{4}$ 倍, 因此如何快速搜索确定伪码相位, 是其中需要解决的关键问题.

长码捕获按照信号处理方式不同分为: 时域处理方法 (包括多相关器并行法和匹配滤波法 ${ }^{[1,2]}$ 等) 和频域处理方法 (包括基于 FFT 的捕获方法 ${ }^{[3,4]}$ ). 为了减小搜索的相位数, 采用多点重叠的原理 一次搜索多个相位, 这类方法包括平均搜索法 ${ }^{[5]}$ 和扩展复制重叠 XFAST (extended replica folding acquisition search technique) 算法 $[6 \sim 8]$. 其中时域处理方法单元运算简单, 数据处理实时性要求高, FPGA 和 ASIC 等嵌入式器件基于大量的运算单元, 并行进行乘累加计算, 实现方法中时序要求高, 控 制逻辑复杂. 频域处理方法算法复杂, 对数据批处理操作, DSP 和 CPU 等处理器利用 C 语言和丰富 的库函数, 通过软件实现复杂的处理算法, 但是 DSP 等嵌入式处理器 FFT 处理点数受限, 因而不能 对长码直接进行 FFT 搜索, 并且 FFT 捕获方法捕获时间受处理器主频等限制.

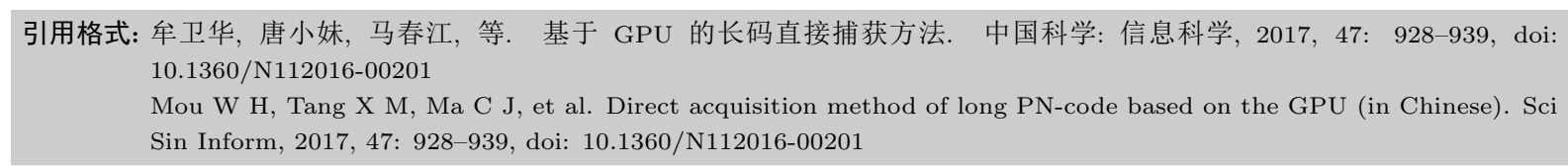




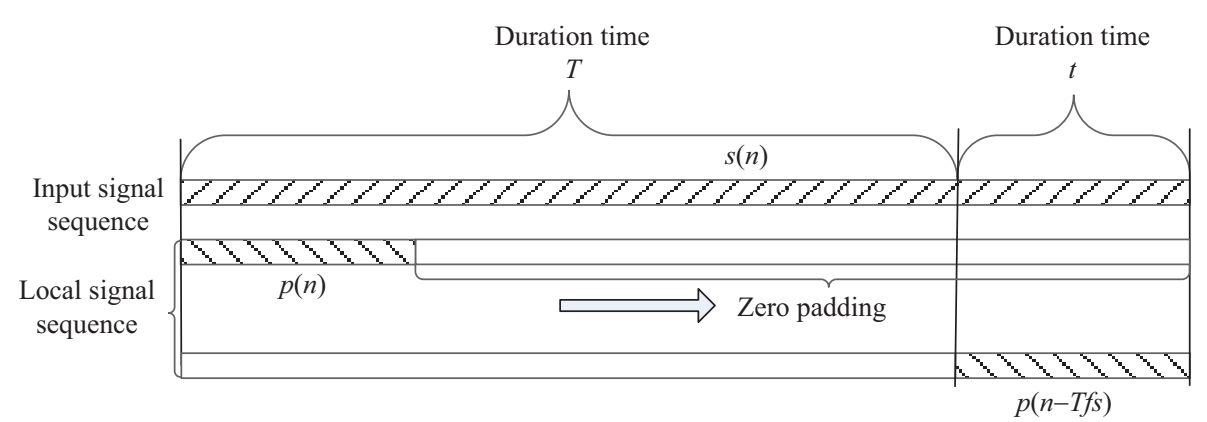

图 1 局部相关码相位空间搜索示意图

Figure 1 Part correlation code phase space searching method

GPU 中有大量的浮点运算单元, 通过利用数以百计的处理器核心, GPU 计算 FFT 的速度可提升 10 倍. 近几年, 基于 GPU 的通用计算技术开始应用于工程计算领域, 相关专家和研究机构对 GPU 通 用计算在导航信号接收领域的应用进行了部分研究 ${ }^{[9 \sim 13]}$, 对基于 GPU 的捕获研究主要集中在短码捕 获分析上, 还未见相关材料对基于 GPU 的长码捕获进行研究.

本文分析了局部相关的码相位空间捕获模型, 为了能够对数据处理任务并行处理, 提出了一种分 段部分重叠局部相关捕获方法, 给出了基于局部相关的全局码相位空间直接捕获和分段捕获的 GPU 实现方法, 并对 GPU 和 CPU 实现方法进行了性能对比验证.

\section{2 基于局部相关的长码时域捕获}

\section{1 基于局部相关的长码全局直接捕获}

对于长周期伪码, 以 GPS P 码为例, 伪码周期为 7 天, 在整个捕获处理时间段内伪码不重复, 不 能像短码一样进行整个伪码周期的相关运算. 基于局部相关的长码相位空间搜索是生成一小段本地参 考信号, 与整个不确定范围内的输入信号序列进行相关运算, 寻找相关最大值从而确定长码相位 ${ }^{[14]}$.

首先, 根据本地时间, 取一段本地长码, 生成采样率为 $f_{\mathrm{s}}$ 、持续时间为 $t$ 的本地信号序列 $p(n)$ 为 $\left\{p(0), p(1), \ldots, p\left(t f_{\mathrm{s}}\right)\right\}$.

将序列 $p(n)$ 补充 $T f_{\mathrm{s}}$ 个 0 , 得到 $p_{0}(n)$, 即

$$
p_{0}(n)= \begin{cases}p(n), & 0 \leqslant n \leqslant t f_{\mathrm{s}}, \\ 0, & t f_{\mathrm{s}}<n \leqslant(T+t) f_{\mathrm{s}} .\end{cases}
$$

记伪码速率 $f_{\mathrm{P}}$, 以采样率 $f_{\mathrm{s}}\left(f_{\mathrm{s}}>2 f_{\mathrm{P}}\right)$ 对信号进行采样, 采集 $T+t$ 秒的输入信号, 剥离载波后 的复信号序列 $s(n)$ 为 $\left\{s(0), s(1), \ldots, s\left[(T+t) f_{\mathrm{s}}\right]\right\}$. 在整个待搜索的相位空间, 共有 $T f_{\mathrm{s}}$ 个可能的伪 码相位值. $s(n)$ 与 $p_{0}(n)$ 的相关值序列 $r(n)$ 计算如 $(2)$ 式:

$$
r(n)=\sum_{k=0}^{(T+t) f_{\mathrm{s}}} s(k) p_{0}(k-n),
$$

其中 $n=0,1, \ldots, T f_{\mathrm{s}}$.

局部相关搜索的示意图如图 1 所示. 


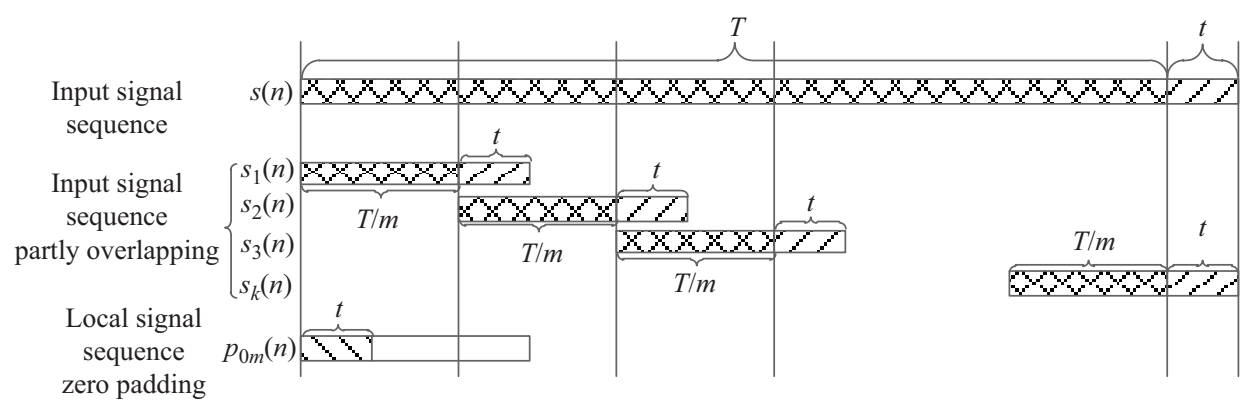

图 2 分段重叠局部相关输入信号划分

Figure 2 Partly overlap segmentation and correlation of input samples

\section{2 利用 FFT 实现线性相关时域捕获}

为了加速相关搜索的速度, 采用 FFT 计算相关值序列 $r(n)$. 短周期伪码扩频信号用 FFT 方法计 算相关值时, 由于伪码的周期重复性, 在处理时间段内的相关搜索为循环相关运算, 可直接应用 FFT 算法. 长周期伪码扩频信号在处理时间段内伪码不重复, 信号相关搜索过程为输入信号与本地信号的 线性相关运算, 需要转化成循环相关后才能应用 FFT 算法. 将 $s(n)$ 的周期拓展序列记为 $\tilde{s}(n), p_{0}(n)$ 的周期拓展序列记为 $\tilde{p}_{0}(n)$, 则 $s(n)$ 与 $p_{0}(n)$ 的循环相关序列 $y_{T+t}(n)$ 计算如 (3) 式:

$$
y_{T+t}(n)=\left[\sum_{k=0}^{(T+t) f_{\mathrm{s}}} \tilde{s}(k) \tilde{p}_{0}[-(n-k)]\right] R_{T+t}(n),
$$

其中 $R_{T+t}(n)$ 为矩形序列, 表示取长度为 $(T+t) f_{\mathrm{s}}$ 的主值序列. 记 $s(n)$ 的离散 Fourier 变换为 $S(k)$, $p_{0}(n)$ 的离散 Fourier 变换为 $P_{0}(k), y_{T+t}(n)$ 的离散 Fourier 变换为 $Y_{T+t}(k)$, 由 (3) 式可得

$$
Y_{T+t}(k)=S(k) P_{0}^{*}(k) .
$$

对频域计算的结果取逆 FFT 可得

$$
y_{T+t}(n)=\operatorname{IFFT}\left[Y_{T+t}(k)\right] .
$$

取循环相关序列 $y_{T+t}(n)$ 的前 $T f_{\mathrm{s}}+1$ 个值, 舍弃后 $t f_{\mathrm{s}}$ 个值, 即得到不确定范围内的相关值序列 $r(n)$.

\section{3 分段部分重叠局部相关捕获}

当伪码相位搜索不确定范围较大时, 需要处理的输入信号序列长度可能超出处理器能力范围, 此 时采用部分重叠分段补零方法, 将输入信号序列部分重叠分段划分, 输入信号序列分段的长度大于本 地信号序列长度即可, 重叠部分持续时间与本地信号序列持续时间相同, 本地信号序列在末尾补零至 输入信号序列分段长度 (zero padding and overlapping). 特别地, 当输入信号序列分段长度为本地信号

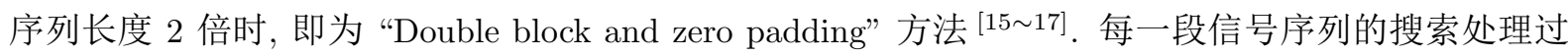
程均采用基于局部相关的搜索方法, 通过 FFT 在频域计算每个分段的相关值, 各段信号搜索处理过程 可利用多核处理器并行进行. 输入信号序列的划分如图 2 所示.

将输入信号序列划分为 $m$ 段, 每一段序列持续时间为 $T / m+t$, 序列重叠时间为 $t$, 则各子序列 $s_{i}(n)$ 为 $\left\{s\left(i T f_{\mathrm{s}} / m\right), s\left(i T f_{\mathrm{s}} / m+1\right), \ldots, s\left[(i+1) T f_{\mathrm{s}} / m+t f_{\mathrm{s}}\right]\right\}$, 其中 $i=0,1, \ldots, m-1$. 
将序列 $p(n)$ 补充 $T f_{\mathrm{s}} / m$ 个 0 , 得到 $p_{0 m}(n)$, 应用上述推导的局部相关码相位空间搜索方法, 计 算出 $s_{i}(n)$ 与 $p_{0 m}(n)$ 的相关值子序列 $y_{i}(n):\left\{y\left(i T f_{\mathrm{s}} / m\right), y\left(i T f_{\mathrm{s}} / m+1\right), \ldots, y\left[(i+1) T f_{\mathrm{s}} / m\right]\right\}$.

所有的 $y_{i}(n)$ 拼接后即可得到整个不确定空间的相关值序列 $r(n)$.

\section{4 运算量与捕获时间分析}

直接 FFT 全局搜索算法中, 输入数据序列 FFT、本地参考序列 FFT 以及结果的 IFFT 再加上 频域复共轭相乘的总计算量为 $O\left(3(T+t) f_{\mathrm{s}} \log (T+t) f_{\mathrm{s}}\right)+5(T+t) f_{\mathrm{s}}$. 分段部分重叠 FFT 搜索算法 中, 输入数据序列 FFT、本地参考序列 FFT 以及结果的 IFFT 再加上频域复共轭相乘的总计算量为 $(2 m+1) \times O\left((T / m+t) f_{\mathrm{s}} \log (T / m+t) f_{\mathrm{s}}\right)+5 m(T / m+t) f_{\mathrm{s}}$. 经过比较, 两种算法总计算量在一个数量 等级, 但分段部分重叠局部相关捕获算法, 将总的数据分段划分进行处理, 从而方便利用多核处理器 并行运算, 提高处理器运算资源利用率.

\section{3 局部相关长码时域捕获的 GPU 实现}

\section{1 全局直接搜索的 GPU 实现}

GPU 中具有数以百计的浮点处理器, 其快速 Fourier 变换库 cuFFT 充分利用大量的处理器资源 进行 FFT 运算, 其 1 维 FFT 变换最大可支持 $128 \mathrm{M}$ 个元素. GPS P 码伪码速率为 $10.23 \mathrm{MHz}$, 假 设采样率为 $37.5 \mathrm{MHz}$, 搜索时间不确定范围为 $\pm 1 \mathrm{~s}$, 则整个待搜索的伪码相位共有 $75 \mathrm{M}$ 个, 不超过 1 维 $\mathrm{FFT}$ 变换的最大数据处理量, 因此可采用 $\mathrm{cuFFT}$ 实现基于局部相关的码相位空间全局直接搜索. 基于 GPU 的全局直接搜索方法步骤如下 ${ }^{[18]}$ :

(1) 根据本地时间 $t_{0}$, 取 $\left[t_{0}, t_{0}+t\right]$ 时间段持续时间为 $t$ 的 $\mathrm{P}$ 码数据, 根据采样率 $f_{\mathrm{s}}$ 生成本地 参考信号序列 $p(n)$;

(2) 以采样率 $f_{\mathrm{s}}$ 采集 $(T+t)$ 秒输入信号 (其中 $T$ 为不确定时间范围), 剥离载波后生成复信号 序列 $s(n)$;

(3) 在 GPU 设备端为输入信号序列 $s(n)$ 和本地参考信号序列 $p(n)$ 分配存储空间, 使用 cud$\mathrm{aMemcpy}()$ 函数将输入信号序列 $s(n)$ 和本地参考信号序列 $p(n)$ 由主机端拷贝到设备端;

(4) 将本地信号序列 $p(n)$ 补充 $T f_{\mathrm{s}}$ 个 0 , 得到序列 $p_{0}(n)$;

(5) 调用函数 cufftPlan1d() 创建 Fourier 变换计划;

(6) 调用函数 cufftExecC2C() 和 cufftExecR2C(), 计算输入信号序列 $s(n)$ 的 Fourier 变换序列 $S(k)$ 和本地信号 Fourier 变换序列 $P_{0}(k)$;

(7) 在 GPU 中逐点计算 $S(k)$ 与 $P_{0}(k)$ 的共轭乘积, 得到序列 $Y_{T+t}(k)$;

(8) 调用函数 cufftExecC $2 \mathrm{C}()$ 计算 $Y_{T+t}(k)$ 的逆 $\mathrm{FFT}$, 得到循环相关序列 $y_{T+t}(n)$, 截取每个相 关序列的前 $T f_{\mathrm{s}}+1$ 个值, 得到整个不确定时间范围内的相关值序列 $r(n)$;

(9) 在 GPU 中逐点计算相关值序列 $r(n)$ 的模值, 得到模值序列 $M(n)$;

(10) 搜索 $M(n)$ 的最大值, 判断是否超过检测门限, 并确定码相位值 $t_{d}$.

\section{2 分段重叠搜索的 GPU 实现}

在 FPGA 和 ASIC 中利用大量硬件乘加器等运算资源并行运算, 能够实现数据的快速 FFT 运算, 目前长码直接捕获主要由 FPGA 和 ASIC 等实现. 在 FPGA 中由于硬件资源的限制, FFT 运算点数 
受限, 在伪码相位不确定度范围较大时, 需要采用重叠分段补零 FFT 方法, 将输入数据重叠分段划分. 为了实现多组不同分段数据的 $\mathrm{FFT}$, 需要严格控制输入和输出数据的分批切换时序以及长码提取的 码相位与时序, 控制逻辑复杂, 仿真验证时间长. 在 cuFFT 函数库中, FFT 变换函数可以同时对输入 的多组不同的数据进行 FFT 批处理操作, 据此能够在 GPU 中实现分段部分重叠的局部相关捕获算 法. 采用软件方法实现多组分段数据的 FFT 批处理运算, 简化了数据分批切换的控制逻辑, 仿真验证 速度快. 但是基于 GPU 的长码直接捕获方法, 需要采用专用硬件将信号与长码采集并传输至运算主 机, 同时需要在主机中增加 GPU 运算卡, 增大了功率消耗.

以 GPS P 码捕获为例, 将基于 GPU 的全局直接搜索方法中步骤 (4) (8) 做如下替换:

(4) 将输入信号序列部分重叠分段划分, 得到 $m$ 个序列 $s_{i}(n), i=0,1, \ldots, m-1$, 将本地信号序 列 $p(n)$ 补充 $T f_{\mathrm{s}} / m$ 个 0 , 得到序列 $p_{0 m}(n)$;

(5) 调用函数 cufftPlan1d() 和 cufftPlanMany() 创建 Fourier 变换计划;

(6) 调用函数 cufftExecC2C() 和 cufftExecR2C(), 批处理计算分段划分的 $m$ 个序列 $s_{i}(n)$ 的 Fourier 变换序列 $S_{i}(k)$ 和本地信号 Fourier 变换序列 $P_{0 m}(k)$;

(7) 在 GPU 中逐点计算 $S_{i}(k)$ 与 $P_{0 m}(k)$ 的共轭乘积, 得到序列 $Y_{i m}(k)$;

(8) 调用函数 cufftExecC2C() 计算 $Y_{i m}(k)$ 的逆 $\mathrm{FFT}$, 得到 $m$ 个循环相关序列 $y_{i m}(n)$, 截取每个 相关序列的前 $T f_{\mathrm{s}} / m$ 个值 $y_{i}(n)$, 拼接后得到整个不确定时间范围内的相关值序列 $r(n)$.

分段重叠搜索方法将输入数据进行划分, 降低了数据处理的规模和存储要求, 适应于不同性能的 GPU 实现, 对数据的划分同时有利于多核处理器对数据进行并行任务处理.

\section{4 长码时频搜索策略及检测性能}

在基于 FPGA 的长码直接捕获方法中, 为了在时域和频域并行搜索伪码相位和 Doppler 频率, 通 常采用部分匹配滤波器 PMF 和 FFT 结合的实现架构, 其中匹配滤波器部分采用流水并行机制在时 域搜索伪码相位, 通过增加硬件资源实现滤波器的并行乘加运算和流水处理机制, 能够在每个数据点 实时计算, 完成该点的时频二维搜索处理, FFT 模块处理多段匹配滤波器输出的部分相关值并行搜索 Doppler, 该架构易于 FPGA 实现 ${ }^{[19]}$.

基于 GPU 的长码时频二维直接捕获把多段匹配滤波器码相位搜索部分采用 FFT 算法实现, 将 分段的输入数据和本地长码信号通过 FFT 变换到频域, 在频域通过共轭乘实现相干计算并行搜索伪 码相位, 对于 Doppler 频率的搜索, 利用小点数 FFT 处理部分相关值估计得到. 对于 Doppler 频率搜 索, 还可以通过移位频域序列得到不同频偏的本地参考信号, 与输入信号频域序列相乘的方法来实现.

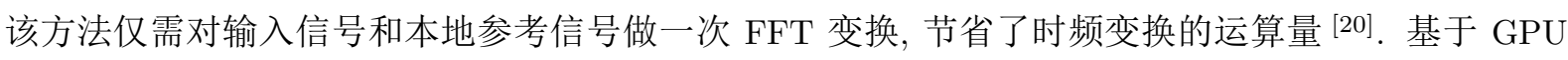
的长码直接捕获方法运用 FFT 算法压缩相关运算量, 通过增大算法复杂度获得相关运算的并行度, 适 于软件接收机实现.

假定虚警概率 $P_{\mathrm{fa}}=10^{-8}$, 则由 (6) 式:

$$
V_{\mathrm{t}}=\sigma_{\mathrm{n}} \sqrt{-2 \ln P_{\mathrm{fa}}}
$$

得到判决门限 $V_{\mathrm{t}}$ 为 $6.07 \sigma_{\mathrm{n}}$, 式中 $\sigma_{\mathrm{n}}$ 为噪声均方根值. 相关检测性能仿真如图 3 所示, 当虚警概率 $P_{\mathrm{fa}}=10^{-8}$, 检测概率 $P_{\mathrm{d}}=0.995$ 时, 检测判决处需要的最小信噪比 $S / N$ 约 $15.3 \mathrm{~dB}$.

载噪比 $C / N_{0}$ 与信噪比关系如 (7) 式:

$$
C / N_{0}=S / N-10 \log (t),
$$




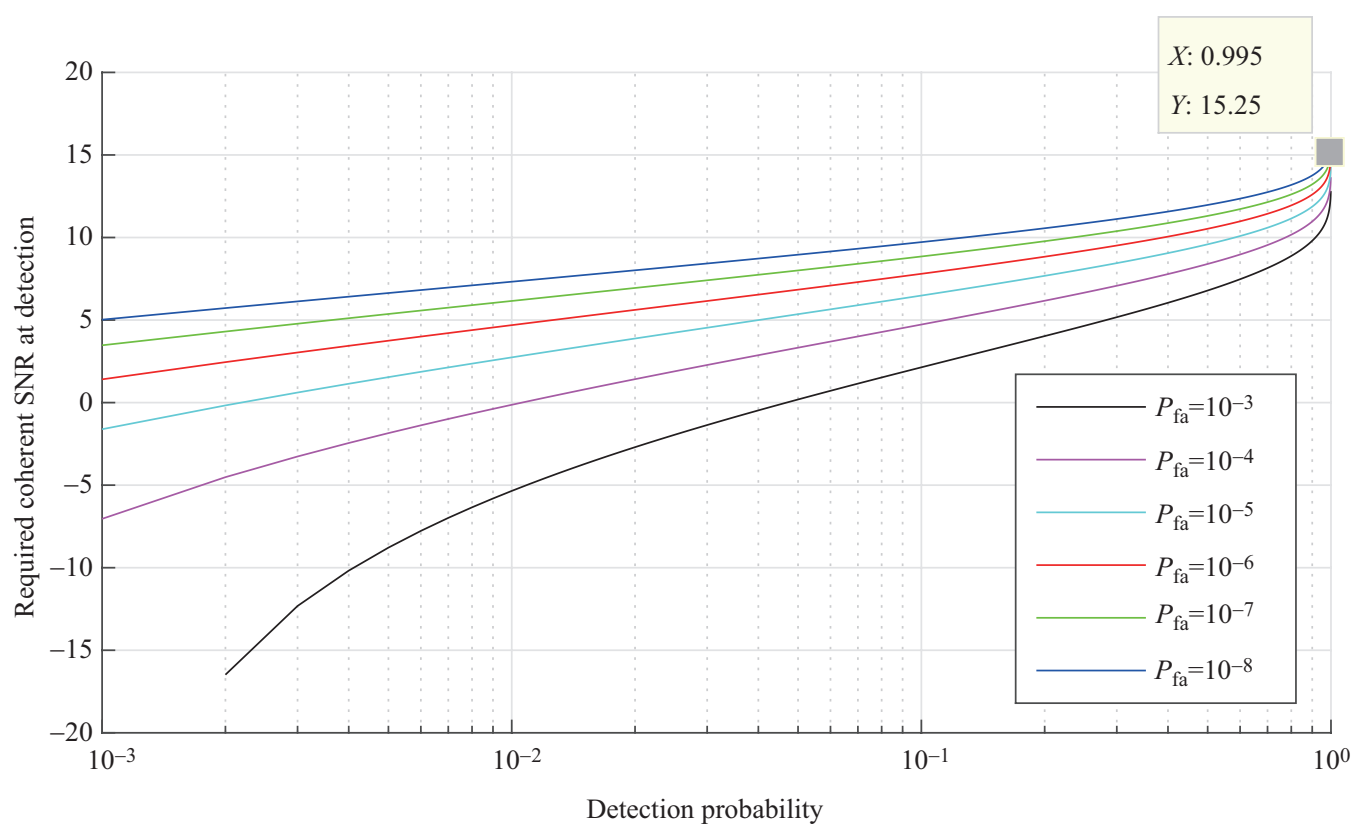

图 3 相干检测性能曲线

Figure 3 Performance of coherent detection

式中 $t$ 表示相干积分时间, 当 $t$ 取 $1 \mathrm{~ms}$ 时, 达到检测性能需要的最小载噪比 $C / N_{0}$ 需大于 $45.3 \mathrm{~dB} \cdot \mathrm{Hz}$.

当存在 Doppler 误差 $f_{\mathrm{e}}$ 时, 由 (8) 式算得相关损耗:

$$
L=10 \log \left[\sin c\left(\pi \cdot f_{\mathrm{e}} \cdot t\right)\right]^{2} .
$$

由 (7) 和 (8) 式, 在 Doppler 范围 $[-5 \mathrm{kHz}, 5 \mathrm{kHz}]$ 内, 相关损耗不超过 $1 \mathrm{~dB}$, 本地参考信号序列持续 时间 $t$ 需取 $0.05 \mathrm{~ms}$, 为达到检测性能输入信号 $C / N_{0}>58.3 \mathrm{~dB} \cdot \mathrm{Hz}$, 此时利用上述算法搜索确定伪码 相位后, 再利用一组分段相关值序列进行 Doppler 频率估计. 为了提高频率估计的准确度, 将分段相关 值序列后补零, 对补零序列 FFT 变换估计出信号 Doppler 初始值 ${ }^{[21]}$. 当输入信号 $C / N_{0}>45.3 \mathrm{~dB} \cdot \mathrm{Hz}$ 时, 本地参考信号序列持续时间 $t$ 取 $1 \mathrm{~ms}$, 相关损耗不超过 $1 \mathrm{~dB}$, Doppler 容限范围为 $[-250 \mathrm{~Hz}$, $250 \mathrm{~Hz}$. 为了覆盖整个 Doppler 不确定范围, 需要利用频域移位的方法将步骤 $(6)$ 中的 $P_{0}(k)$ 和 $P_{0 m}(k)$ 依次移位补偿搜索 Doppler, 重复步骤 $(7) \sim(10)$ 搜索 21 个 Doppler 槽, 从而完成长码的时频 二维搜索 ${ }^{[22]}$.

在导航卫星仰角较低或导航信号被高楼、树叶等遮挡时, 信号相比正常情况下更弱, 为了能够捕 获到信号, 需要进一步增大积分时间来提高捕获灵敏度. 由于 Doppler 和信息边界的存在, 相干积分 时间增大到一定程度后，只能通过非相干累加的方式来增大积分时间. 码 Doppler 会引起码片滑动, Doppler 或积分时间较大时, 码片滑动超过一个码片后, 再增大积分时间反而会恶化捕获性能, 此时 需要补偿伪码 Doppler, 然后基于 GPU 计算长码分段相关值后再进行非相干累加 ${ }^{[23]}$. 在虚警概率 $P_{\mathrm{fa}}=10^{-8}$, 检测概率 $P_{\mathrm{d}}=0.995$ 时, 非相干累加增益仿真如图 4 所示 ${ }^{[24]}$.

从图 4 中可以看出, 最初累加增益明显, 随着累加次数增大, 增益逐渐趋缓. 取非相干累加 200 次, 增益约为 $17.0 \mathrm{~dB}$, 此时为达到检测指标, 在不计入损耗情况下, 信号载噪比 $C / N_{0}$ 仅需大于 $28.3 \mathrm{~dB} \cdot \mathrm{Hz}$. 


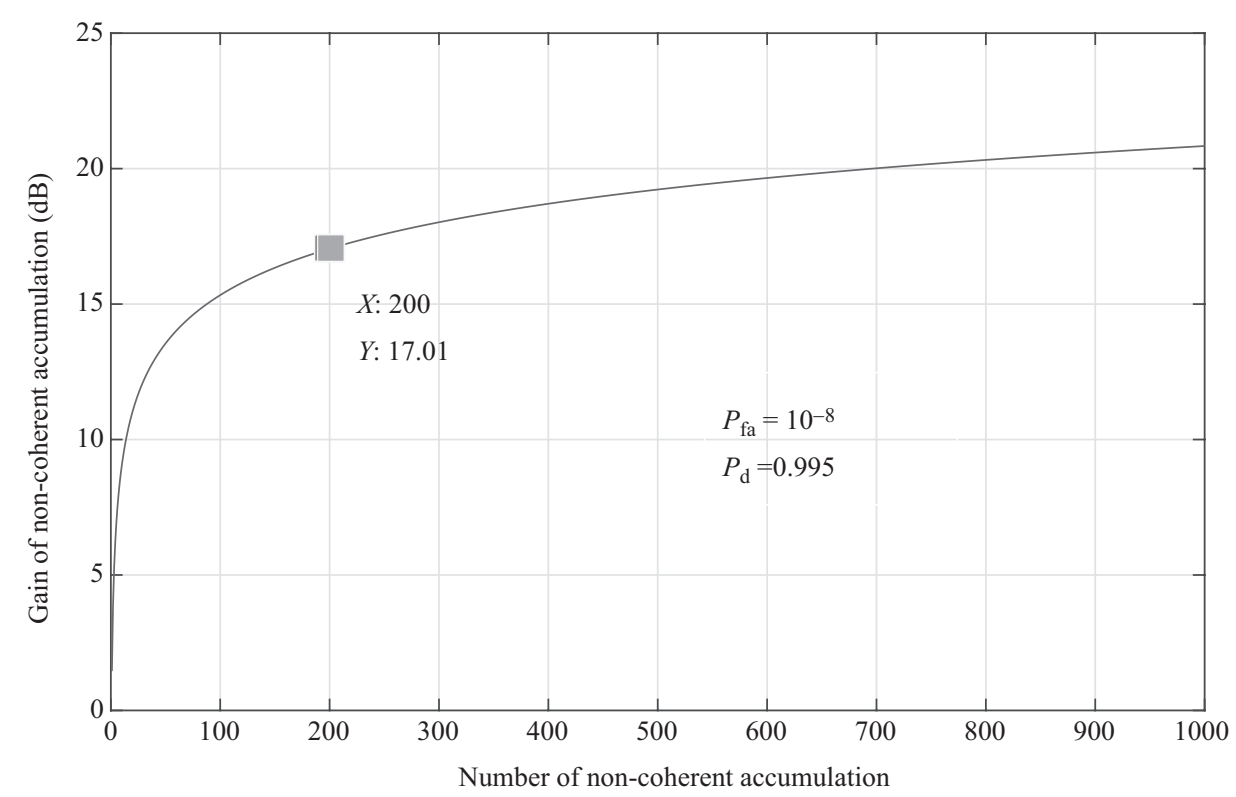

图 4 非相干累加增益曲线

Figure 4 Gain of non-coherent accumulation

表 1 长码直接捕获 CPU 与 GPU 实现耗时

Table 1 Time consuming of implementation based on CPU and GPU

\begin{tabular}{ccc}
\hline & $\begin{array}{c}\text { Direct global } \\
\text { searching algorithm }\end{array}$ & $\begin{array}{c}\text { Partly overlap segmentation } \\
\text { searching algorithm }\end{array}$ \\
\hline Time consuming of CPU implementation (ms) & 17600.3 & 4759.3 \\
Time consuming of GPU implementation (ms) & 322.4 & 285.3 \\
Acceleration rate & 54.6 & 16.7 \\
\hline
\end{tabular}

\section{5 算法测试验证}

测试条件与实验环境如下: 信号采样率为 $37.5 \mathrm{MHz}$, 时间不确定范围为 $\pm 1 \mathrm{~s}$, 频率不确定范围为 $\pm 5 \mathrm{kHz}$. 在 GPU 上调用 cuFFT 函数库实现上述全局直接捕获算法与分段捕获算法, 与 $\mathrm{CPU}$ 上采用 FFTW 函数库的实现进行捕获时间对比, 分析 GPU 实现的长码捕获方法的性能. 仿真验证平台 CPU 为 Intel 32 核 E5-2640, 主频 $2.6 \mathrm{GHz}, \mathrm{GPU}$ 为 Tesla K80.

\section{1 长码直接捕获 CPU 与 GPU 实现对比测试}

对于 GPU 实现的捕获核函数耗时, 采用 Nsight Performance Analysis 进行测试. Windows 属于抢 占式操作系统, 线程并非独占 $\mathrm{CPU}$, 为了得到 CPU 实现的捕获算法线程耗时, 采用 QueryThreadCycleTime() 函数, 通过该函数能够计算得到捕获算法线程获得的 CPU 时间, 而不计入线程被调度程序 暂停中断的时间 ${ }^{[25]}$.

为了对比捕获速度, 测试在 1 个频率槽下对所有不确定长码相位空间搜索的耗时, CPU 与 GPU 实现的捕获耗时如表 1 所示. 
表 2 GPU 长码直接捕获算法各阶段耗时

Table 2 Each stage time consuming of direct acquisition algorithm based on GPU

\begin{tabular}{ccc}
\hline Stage of algorithm & $\begin{array}{c}\text { Direct global } \\
\text { searching algorithm }(\mathrm{ms})\end{array}$ & $\begin{array}{c}\text { Partly overlap segmentation } \\
\text { searching algorithm }(\mathrm{ms})\end{array}$ \\
\hline Zero padding and overlapping & 19.6 & 44.9 \\
FFT of input signal & 84.3 & 72.6 \\
FFT of local signal & 84.2 & 0.1 \\
Conjugate multiplication & 32.8 & 71.4 \\
IFFT & 84.6 & 73.0 \\
Modulus & 14.3 & 20.7 \\
Signal detection & 2.6 & 2.6 \\
Total time consuming & 322.4 & 285.3 \\
\hline
\end{tabular}

从表 1 可以看出, 长码直接捕获 GPU 实现相比 CPU 实现, 不论全局直接搜索还是分段重叠搜索, 运算速度均进一步提升, 其中全局直接搜索 GPU 实现方法速度提高了 54.6 倍, 分段重叠搜索 GPU 实现方法速度提高了 16.7 倍.

将第 2 节中基于局部相关的长码全局直接捕获方法和分段部分重叠局部相关捕获方法在 GPU 上 实现, 首先在 GPU 中将输入数据分段划分、本地数据补零, 然后分别对输入数据和本地数据做 FFT 变换, 对应共轭相乘后做 IFFT 变换, 取复相关序列模值得到幅值大小, 进行信号检测后得到捕获结 果. 各个阶段耗时如表 2 所示.

从表 2 可以看出, GPU 上实现的分段重叠捕获方法耗时相比全局直接捕获方法耗时稍小, 但分段 重叠捕获方法中重叠冗余数据划分、共轭乘以及取模值运算多消耗的时间, 抵消了本地参考信号 FFT 时间及算法的部分优势.

当输入信号 $C / N_{0}>45.3 \mathrm{~dB} \cdot \mathrm{Hz}$ 时, 本地参考信号序列持续时间 $t$ 取 $1 \mathrm{~ms}$, 将整个 $\pm 5 \mathrm{kHz}$ 频率不 确定范围划分为间隔为 $500 \mathrm{~Hz}$ 共 21 个频率槽, 最大的搜索时间需要再增加 20 个共轭乘、IFFT、取模 值和信号检测的时间, 根据表 2 中的测试数据, 采用全局直接搜索的最大捕获时间为 $322.4+20 \times(32.8+$ $84.6+14.3+2.6)=3008.4(\mathrm{~ms})$. 采用分段重叠搜索的最大捕获时间为 $285.3+20 \times(71.4+73.0+20.7+2.6)$ $=3639.3(\mathrm{~ms})$.

\section{2 捕获结果}

为测试 GPU 捕获的结果, 设置信号参数如下: 输入信号码相位偏移相对本地参考信号偏移 15000001 个采样点, 信号 Doppler 测试 $0 \mathrm{~Hz}$ 和 $5 \mathrm{kHz}$ 两种情况, 本地参考信号持续时间取 $0.05 \mathrm{ms,}$ 利用 GPU 并行计算搜索整个码相位不确定空间, 捕获结果如图 5 所示.

从图 5 中可以看出, Doppler 为 $0 \mathrm{~Hz}$ 和 $5 \mathrm{kHz}$ 时, 在采样点偏移 15000001 处都可以正确检测出 相关峰, Doppler 为 $5 \mathrm{kHz}$ 时, 相关峰略有损耗.

下面测试 Doppler 频率估计结果, 在搜索出正确的码相位后, 再计算 100 个分段相干积分长度为 $0.1 \mathrm{~ms}$ 的相关值得到一个序列, 将该分段相关值序列后补零成 1024 点, 对序列 FFT 变换估计信号 Doppler, 频率估计误差最大为 $\frac{10 \times 10^{3}}{2 \times 1024} \approx 4.88(\mathrm{~Hz})$. 信号 Doppler 为 $100 \mathrm{~Hz}$ 时, 算法测试的结果如 图 6 所示. 

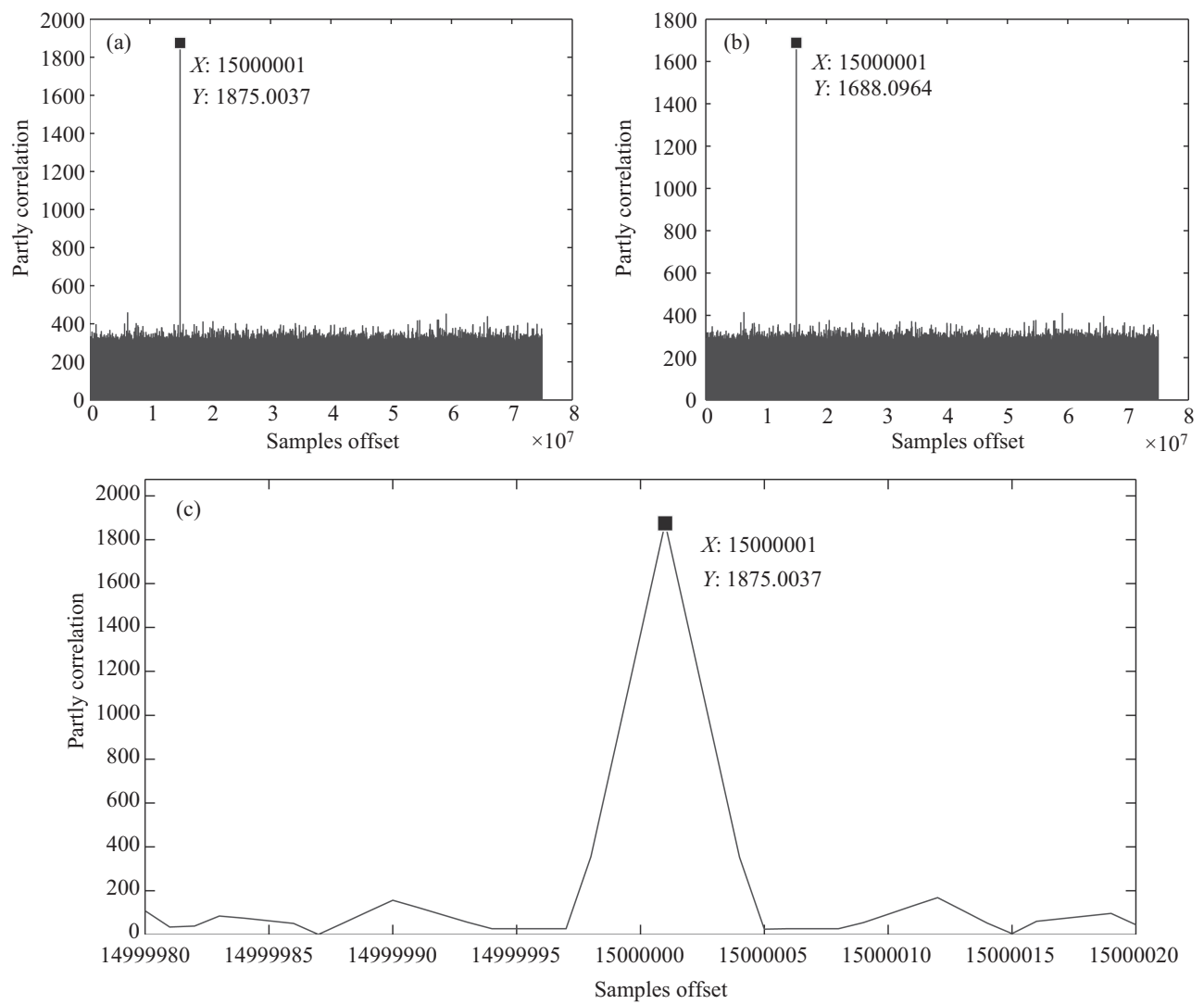

图 5 基于 GPU 的局部相关码相位捕获结果

Figure 5 Code phase acquisition results based on GPU. (a) Samples offset: 15000001, Doppler frequency: $0 \mathrm{~Hz}$; (b) samples offset: 15000001, Doppler frequency: $5 \mathrm{kHz}$; (c) zoom in (a) at 15000000 samples offset

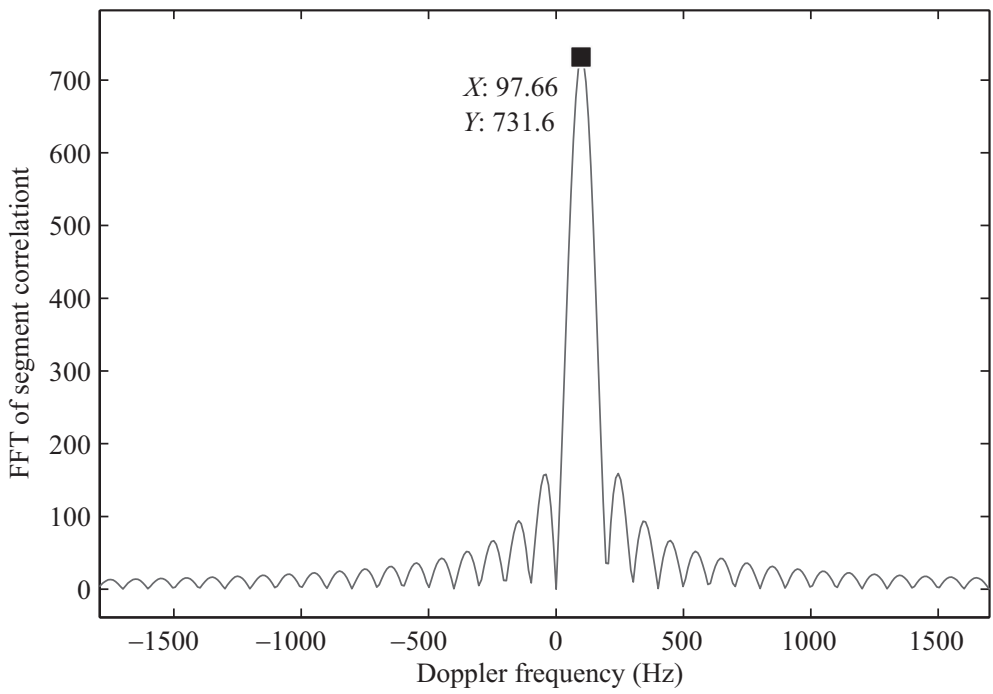

图 6 利用分段相关值估计 Doppler 频率

Figure 6 Doppler frequency estimation based on segment correlation 


\section{6 结束语}

针对长周期伪码直接捕获时大量码相位搜索问题, 提出了一种基于 GPU 的局部相关时域搜索方 法, 突破了嵌入式器件 FFT 变换处理点数限制. 利用 GPU 中数以百计的浮点运算单元并行进行 FFT 运算, 直接对长周期伪码扩频信号不确定范围内的伪码相位全局搜索, 分段重叠局部相关时域搜索, 对 输入数据分段划分, 可以充分发挥多核的并行处理优势. 相比基于 CPU 的长码直接捕获方法, 基于 GPU 的长码直接捕获方法速度显著提高. 该方法与平均搜索法、XFAST 等方法结合使用可以进一步 提高搜索速度. 该方法提高了长码直接捕获的实时性, 实现了基于 GPU 的长码直接时域捕获, 相比在 FPGA 中实现的方法, 修改灵活方便, 可广泛应用于信号监测接收和中心站信号处理的长码直接捕获.

随着 GPU 运算能力的进一步增强, 基于 GPU 能够在频域搜索更多的频率单元, 能够进行更长时 间的非相干累加, 从而能够处理更大动态和更低信噪比的信号, 提高系统处理性能指标.

\section{参考文献}

$1 \mathrm{Wu} \mathrm{H} \mathrm{B,} \mathrm{Hu} \mathrm{Y} \mathrm{H,} \mathrm{He} \mathrm{Z} \mathrm{M,} \mathrm{et} \mathrm{al.} \mathrm{Design} \mathrm{and} \mathrm{implementation} \mathrm{of} \mathrm{high} \mathrm{dynamic} \mathrm{P-code} \mathrm{direct} \mathrm{acquisition} \mathrm{based} \mathrm{on}$ FPGA. GNSS World China, 2010, 35: 7-11 [吴华兵, 胡永辉, 何在民, 等. 基于 FPGA 的高动态 P 码直捕算法设计 与实现. 全球定位系统, 2010, 35: 7-11]

2 Qin Y, Zhang B N, Guo D S, et al. Research of P-code direct acquisition technology at low signal-to-noise rate in high dynamic condition. J Astronautics, 2009, 30: 760-764 [秦勇, 张邦宁, 郭道省, 等. 低信噪比高动态条件下 $\mathrm{P}$ 码直接 捕获技术研究. 宇航学报, 2009, 30: 760-764]

3 Zhang B, Yang C, Xie N, et al. Application of fast FFT acquisition algorithm in GPS C/A and P(Y) codes. Telecommun Eng, 2008, 48: 34-38 [张博, 杨春, 解楠, 等. FFT 快速捕获算法在 GPS C/A 码与 P(Y) 码中的应用. 电讯技术, 2008, 48: 34-38]

4 Wang J, Li J Q, Wu S L. P-code direct acquisition algorithm and it's resource optimization method. J Astronautics, 2011, 32: 2491-2498 [王俊, 李加琪, 吴嗣亮. P 码直接捕获算法的设计及资源优化. 宇航学报, 2011, 32: 2491-2498]

5 Zhang L, Chu H B, Zhang N T. Research on fast direct acquisition method of satellite navigation signal. J Commun, 2007, 28: 120-124, 129 [张琳, 初海涁, 张乃通. 卫星导航信号长码快速直接捕获算法研究. 通信学报, 2007, 28: 120-124, 129]

6 Yang C, Vasquez J, Chaffee J. Fast direct P(Y)-code acquisition using XFAST. In: Proceedings of the 12th International Technical Meeting of the Satellite Division of the Institute of Navigation, Nashville, 1999. 317-324

7 Tang X M, Yong S W, Wang F X. Performance of XFAST in the presence of code Doppler. J Commun, 2010, 31: 54-59 [唐小妹, 雍少为, 王飞雪. 存在伪码多普勒条件下的 XFAST 性能分析. 通信学报, 2010, 31: 54-59]

8 Tang X M, Pang J, Huang Y B, et al. Optimization of XFAST design. J Cent South Univ (Sci Tech), 2014, 45: 1113-1118 [唐小妹, 庞晶, 黄仰博, 等. XFAST 长码直捕算法参数优化设计. 中南大学学报 (自然科学版), 2014, 45: 1113-1118]

9 Yang J, Liu Y F. Parallel-acquisition of GPS signal based on graphic processing unit. J Chinese Inertial Tech, 2012, 30: 430-434 [杨静, 刘一霏. 基于 GPU 的 GPS 信号并行捕获. 中国惯性技术学报, 2012, 30: 430-434]

10 Park K W, Yang J S, Park C, et al. Implementation of GPGPU based real-time signal acquisition and tracking module for multi-constellation GNSS software receiver. In: Proceedings of the 27th International Technical Meeting of the Satellite Division of the Institute of Navigation, Tampa, 2014. 1410-1416

11 Huang B, Yao Z, Guo F, et al. STARx - a GPU based multi-system full-band real-time GNSS software receiver. In: Proceedings of the 26th International Technical Meeting of the Satellite Division of the Institute of Navigation, Nashville, 2013. 1549-1559

12 Pany T, Riedl B, Winkel J. Efficient GNSS signal acquisition with massive parallel algorithms using GPUs. In: Proceedings of the 23rd International Technical Meeting of the Satellite Division of the Institute of Navigation, Portland, 2010. 1889-1895

13 Wang K D, Li H T, Hou S D, et al. Implementation of a GPS signal FFT acquisition algorithm with a GPU chip. GNSS World China, 2011, 36: 12-16 [王可东, 李鸿田, 侯绍东, 等. GPS 信号 FFT 捕获的 GPU 实现. 全球定位系 
统, 2011, 36: 12-16]

14 Chun Y. Sequential block search for direct acquisition of long codes under large uncertainty. In: Proceedings of the 14th International Technical Meeting of the Satellite Division of the Institute of Navigation, Long Beach, 2001. 408-414

15 Lin D M, Tsui J B Y, Howell D. Direct P(Y)-code acquisition algorithm for software GPS receivers. In: Proceedings of the 12th International Technical Meeting of the Satellite Division of the Institute of Navigation, Nashville, 1999. 363-368

16 Yu G Q, Jin R, Li Y W. Principle and implement of P-code direct acquisition based on average block zero padding. J Hebei Univ Sci Tech, 2014, 35: 172-178 [于国庆, 靳荵, 李永伟. 均值分组块补零 P 码直捕算法原理及实现. 河北科 技大学学报, 2014, 35: 172-178]

17 Zhang X B, Zhang Y, Liu T. Research on GPS long-code-acquisition method. J Univ Electron Sci Tech China, 2008, 37: 62-65 [张新波, 张扬, 刘田. GPS 接收机 $\mathrm{P}(\mathrm{Y})$ 直捕方法研究. 电子科技大学学报, 2008, 37: 62-65]

18 Chen Y, Wang Y Q, Liu Y. Acquisition method with spread spectrum technique based on GPU acceleration. J Grad Univ Chinese Acad Sci, 2012, 29: 240-245 [陈源, 王元钦, 刘莹. 基于 GPU 加速的扩频信号捕获方法. 中国科学院 研究生院学报, 2012, 29: 240-245]

19 Wu G J, Zou Y Z, Tan M Q. A novel acquisition algorithm based on parallel search in time and frequency domain and FPGA implementation. J Beijing Univ Posts Telecommun, 2009, 32: 91-95 [吴高进, 邹永忠, 谭敏强. 时频二维搜索 捕获算法及其 FPGA 实现. 北京邮电大学学报, 2009, 32: 91-95]

20 Tian M H, Feng Y X, Pan C S. Research of a method of direct acquisition based on P code in frequency domain. ACTA Electron Sin, 2007, 35: 549-552 [田明浩, 冯永新, 潘成胜. 一种基于 P 码的频域直接捕获算法的研究. 电子 学报, 2007, 35: 549-552]

21 Wolfert R, Chen S, Kohli S, et al. Rapid direct P(Y)-code acquisition in a hostile environment. In: Proceedings of the 11th International Technical Meeting of the Satellite Division of the Institute of Navigation, Nashville, 1998. 353-360

22 Kaplan E D, Hegarty C J. Understanding GPS: Principles and Applications. 2nd ed. Beijing: Publishing House of Electronics Industry, 2012. 162-166 [Kaplan E D, Hegarty C J. 寇艳红, 译. GPS 原理与应用 (第二版). 北京: 电子 工业出版社, 2012. 162-166]

23 Li H, Zhou H, Lu M Q, et al. Code-doppler-compensation based direct acquisition method for weak GNSS long PNcode. In: Proceedings of the 1st China Satellite Navigation Conference, Beijing, 2010. 87-96 [李洪, 周辉, 陆明泉, 等. 基于码多普勒补偿的微弱 GNSS 长码快速直接捕获算法. 第一届中国卫星导航学术年会, 北京, 2010. 87-96]

24 Li J, Liu F, Long T. Direct acquisition of GPS P-code under low-SNR. Trans Beijing Inst Tech, 2009, 29: 68-72 [李 健, 刘峰, 龙腾. 低信噪比下 GPS 信号 P 码直接捕获技术. 北京理工大学学报, 2009, 29: 68-72]

25 Jeffrey R, Christophe N. Windows via C/C++. 5th ed. Beijing: Tsinghua University Press, 2008. 170-174 [Jeffrey R, Christophe N. 葛子昂, 周靖, 廖敏, 译. Windows 核心编程 (第 5 版). 北京: 清华大学出版社, 2008. 170-174] 


\title{
Direct acquisition method of long PN-code based on the GPU
}

\author{
Weihua MOU ${ }^{1,2^{*}}$, Xiaomei TANG ${ }^{1}$, Chunjiang $\mathrm{MA}^{1}$, Gang $\mathrm{OU}^{1,2}$ \& Feixue WANG ${ }^{1,2}$ \\ 1. College of Electronic Science and Engineering, National University of Defense Technology, Changsha 410073, \\ China; \\ 2. BeiDou Satellite Navigation Product 2301 Quality Testing Center, Changsha 410073, China \\ * Corresponding author. E-mail: drmou@163.com
}

\begin{abstract}
In order to solve the acquisition problem of long PN-code spread spectrum signals, the partly correlation code phase-space acquisition model and implementation method of the FFT are analyzed. In order to segment data processing tasks and make full use of GPU to speed up FFT, a partly overlapped segmentation acquisition method of long PN-code is researched. Implementations of a direct global searching method and a partly overlapped segmentation searching method based on the GPU are then proposed, which parallel search for code phase using the FFT method, and accelerate FFT using the GPU. Simulation results indicate that, compared to a CPU implementation method, the search speed of the direct acquisition method of long PN-code based on the GPU is improved significantly.
\end{abstract}

Keywords GPU, parallel computing, long PN-code, direct acquisition, FFT

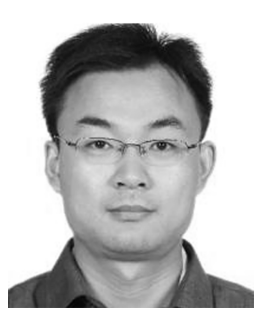

Weihua MOU was born in 1979. He is a Ph.D. candidate at the National University of Defense Technology. $\mathrm{He}$ is an associate professor at the College of Electronic Science and Engineering at the National University of Defense Technology. His main research interests include GPU parallel computing, GNSS signal simulation, and receiver development.

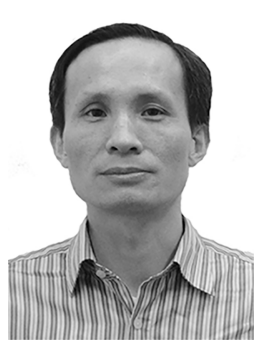

Gang OU was born in 1969. He received a Ph.D. degree from the National University of Defense Technology, Changsha in 1993. He is a professor and Ph.D. supervisor at the College of Electronic Science and Engineering at the National University of Defense Technology. His main research interests include GNSS system simulation, test and assessment technology, and receiver development.

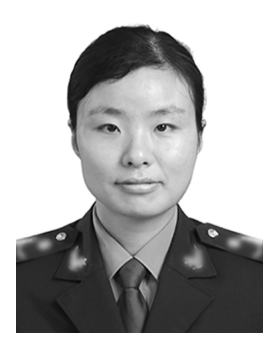

Xiaomei TANG was born in 1982 She received a Ph.D. degree from the National University of Defense Technology, Changsha in 2010. She is an associate professor at the College of Electronic Science and Engineering at the National University of Defense Technology. Her main research interests include GNSS signal processing and receiver development.

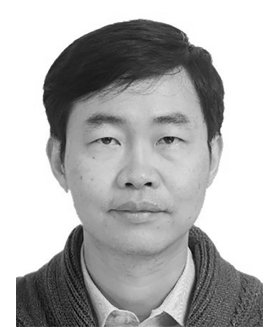

Feixue WANG was born in 1971. He received a Ph.D. degree from the National University of Defense Technology, Changsha in 1998. He is a professor and $\mathrm{Ph} . \mathrm{D}$. supervisor at the College of Electronic Science and Engineering at the National University of Defense Technology. His main research interests include GNSS system technology, GNSS signal processing, and receiver development. 